\title{
A HIAI-LIN TYPE LOG-MAJORIZATION VIA BLOCK-MATRICES
}

\author{
JIAN SHI
}

Abstract. A Hiai-Lin type log-majorization, which is a simultaneous extension of [3, Corollary 3.1] and [4, Corollaries 3.3 and 3.4], is obtained via a block-matrix technique.

Mathematics subject classification (2010): 47A63.

Keywords and phrases: Hiai-Lin type inequality, log-majorization, block-matrix.

\section{REFERENCES}

[1] R. Bhatia, Positive definite matrices, Princeton University Press, 2007.

[2] F. HiAI, M. Lin, On an eigenvalue inequality involving the Hadamard product, Linear Algebra Appl., 515 (2017), 313-320.

[3] R. Lemos, G. SoAres, Some log-majorizations and an extension of a determinantal inequality, Linear Algebra Appl., 547 (2018), 19-31.

[4] J. SHI, Extensions of Hiai-Lin type eigenvalue inequality, Oper. Matrices, 13 (2019), 203-208. 